\title{
Introduction of a portable ultrasound unit into the health services of the Lugufu refugee camp, Kigoma District, Tanzania
}

\author{
David Adler • Katanga Mgalula • Daniel Price • \\ Opal Taylor
}

Received: 1 May 2008 / Accepted: 8 October 2008 / Published online: 14 November 2008

(C) Springer-Verlag London Ltd 2008

\begin{abstract}
Background Diagnostic imaging services are scarce in much of the developing world. Ultrasound is a low-cost, safe, and widely applicable imaging modality.

Aims We delivered a portable ultrasound machine to the Lugufu refugee camp in Tanzania and conducted a course on its use in order to assess the feasibility of introducing this technology into a very low-resource setting.

Methods We conducted an intensive 4-day ultrasound training course for health care providers at Lugufu and established an exam logbook to track the frequency of use, distribution of ultrasound applications, and gender and age distribution of patients.

Results Between 4 October 2005 and 1 November 2007 547 separate ultrasound exams on 460 patients were recorded at Lugufu. Overall, $86 \%$ of patients studied were female. Pregnancy-related exams accounted for $24.1 \%$ of total usage. The 20- to 29-year-old age group accounted for over one third of total exams performed.
\end{abstract}

D. Adler $(\triangle)$

Department of Emergency Medicine, University of Rochester, P.O. Box 655, 601 Elmwood Avenue,

Rochester, NY 14642, USA

e-mail: david_adler@urmc.rochester.edu

\section{K. Mgalula}

Lugufu refugee camp, Tanzania Red Cross,

Kigoma District, Tanzania

D. Price $\cdot$ O. Taylor

Department of Emergency Medicine,

Alameda County Medical Center,

1411 East 31st Street,

Oakland, CA 94602, USA
Conclusion Ultrasound is a feasible and sustainable imaging modality in a very low-resource setting such as the Lugufu refugee camp in Tanzania.

Keywords Ultrasound · Refugee camp · Developing world · Imaging

\section{Introduction}

The availability of diagnostic imaging is very limited in the developing world $[1,2]$. High capital costs, maintenance needs, limited availability of trained personnel and equipment technicians, and austere environmental conditions all contribute to this scarcity of imaging capacity.

The World Health Organization (WHO) has developed a basic radiographic system (BRS) designed to overcome these challenges and increase the availability of X-ray services in developing countries [3]. Still, conventional X-ray equipment is expensive, difficult to repair, immobile, and exposes patients to dangerous radiation.

As an imaging modality, ultrasound has the advantage of being relatively inexpensive, safe [4], mobile, and requires little servicing or installation. Although X-rays are ideal for skeletal imaging, ultrasound is a superior modality for most abdominal, gynecologic, obstetric, vascular, genitourinary, soft tissue, and procedural applications. WHO has recommended the use of ultrasonography in developing countries and has also established minimum specifications for a "general purpose ultrasound scanner" [5-8]. Still, an ultrasound equivalent of the WHO-BRS has yet to be established.

The refugee camp setting is a unique scenario within the developing world that presents even greater barriers to access to diagnostic imaging services. Refugee camps are 
generally remote, poorly accessible by road, and have a severely limited power supply [9]. Need is great, however, as the populations served by refugee camp health services have generally poorer health indicators than the communities from which the refugees originally came [9].

Our review of the literature identified only two reports of introducing general use ultrasonography into a clinical setting in the developing world, both of which were published over 15 years ago and described experiences at major African hospitals in urban areas $[10,11]$. Here we present a feasibility study describing the introduction of a portable ultrasound unit into the health care system of the Lugufu refugee camp in the Kigoma District of Tanzania.

\section{Methods}

Study area and patient population

As an island of relative calm among neighboring nations who have undergone disastrous civil wars over the last decades, Tanzania is both politically and geographically vulnerable to the influx of victims of neighboring wars. The Lugufu refugee camp was established in 1997 by the Tanzanian Red Cross (TRC) as the Great Lakes Crisis in central Africa spread from its start in the summer of 1994.

Lugufu is in the far western region of Tanzania, approximately $90 \mathrm{~km}$ from the shores of Lake Tanganyika. It hosts approximately 90,000 Congolese refugees from a number of tribes (although Bembe predominates) [9]. Female refugees outnumber males (54 vs $44 \%$ ) and the age distribution is skewed sharply to the left with nearly $40 \%$ of the refugees equal to or younger than 15 years old [9]. The Lugufu hospital has individual freestanding burntbrick inpatient wards for women, men, and children. The hospital also has a large outpatient facility with several evaluation rooms. Three health posts are spread among the sprawling camp and offer basic outpatient, laboratory, and pharmacy services. Many important diagnostic tools are lacking, including any form of imaging technology.

Ultrasound training of Lugufu health care providers

Our team delivered a portable ultrasound machine (SonoSite Titan ${ }^{\circledR}$ ) to the health services of the Lugufu refugee camp in autumn 2005. We conducted an intensive 4-day training course with ten members of the health care team at Lugufu. The group consisted of four physicians and six "Clinical Officers" (roughly the equivalent of mid-level practitioners in the USA). Our course emphasized the "goal-oriented" approach of emergency ultrasound. It consisted of morning interactive classroom sessions addressing basic ultrasound physics, knobology, and reviews of specific clinical ultrasound applications including:

- Focused assessment with sonography in trauma (FAST)

- Ultrasound evaluation of the abdominal aorta

- Hepatobiliary ultrasound

- Ultrasound evaluation of pregnancy during the first trimester

- Pregnancy dating and fetal position

- Ultrasound-guided procedures

- Soft tissue ultrasound

- Basic echocardiography

- Renal ultrasound

Afternoons and early evenings were spent conducting hands-on bedside evaluations of inpatients and outpatients at the Lugufu hospital and clinics (Fig. 1). Pathological conditions were plentiful and extemporaneous additions to the training curriculum were made as our group happened upon patients with abdominal masses, thyroid masses, scrotal complaints, and other ultrasonographic findings that were not initially addressed in our didactic sessions (Fig. 2). Although no examination was administered, each of the trainees had at least 20 supervised exams (all applications combined) prior to performing them independently.

Establishment of ultrasound exam logbook

In cooperation with the leadership of the Lugufu health care facilities we established a logbook to track the use of the ultrasound machine. In particular the log was designed to capture data concerning the indication for the ultrasound exam (as determined by the provider), the exam findings, and basic demographic data about the patient. The logbook required exams to be listed as either "positive" or "negative" and had a free space area used to describe the

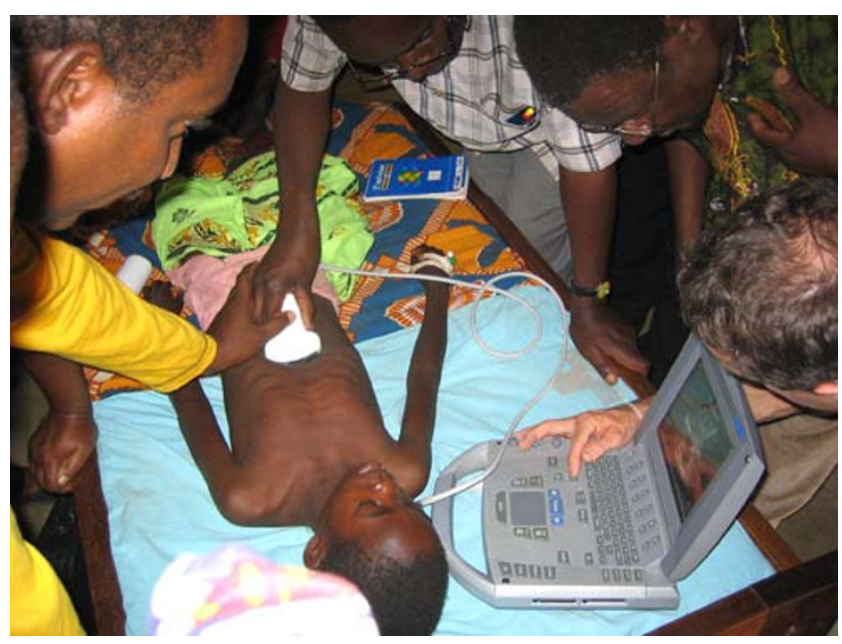

Fig. 1 Hands-on bedside ultrasound training 


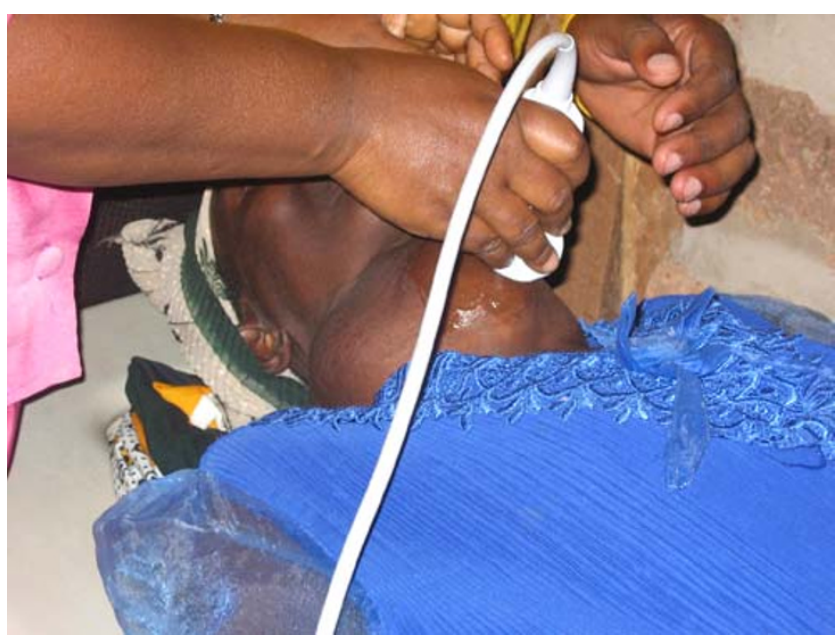

Fig. 2 Examination of a thyroid mass

details of the findings. The free space entries were not standardized for specific findings from specific applications.

Intervention period and follow-up visit

Our first visit to Lugufu was followed by a 2-year period during which the ultrasound machine was left with the health care providers at the refugee camp. The ultrasound $\log$ was maintained locally and periodic updates and $\log$ summaries were sent to us by the TRC. Images were not transmitted to the authors for over-reading during this period.

We revisited Lugufu in 2007 to collect data, assess the status of the ultrasound machine, discuss its use with the local health care providers, and make any necessary adjustments to our effort to make the introduction of the machine a benefit to the refugee population. Instructional sessions were conducted to reinforce prior teaching and bring new health care providers up to date. No proficiency testing or over-reading of saved images was performed.

\section{Results}

During the study period of 4 October 2005 to 1 November 2007, a logbook of ultrasound examinations was maintained at Lugufu. During that period 547 separate ultrasound exams were entered for 460 patients. Among the patients who underwent ultrasound examination 86\% (396/460) were women and $14 \%(64 / 460)$ were men. The age distribution ranged from 3 to 85 years with the majority, $65.8 \%$, ranging from age 20 to 39 years; $36.7 \%$ of the patients were age 20 29 and $29.1 \%$ were age $30-39$ years (Fig. 3).

Although 13 different types of ultrasound studies were recorded in the original data we have consolidated the
Age distribution of patients

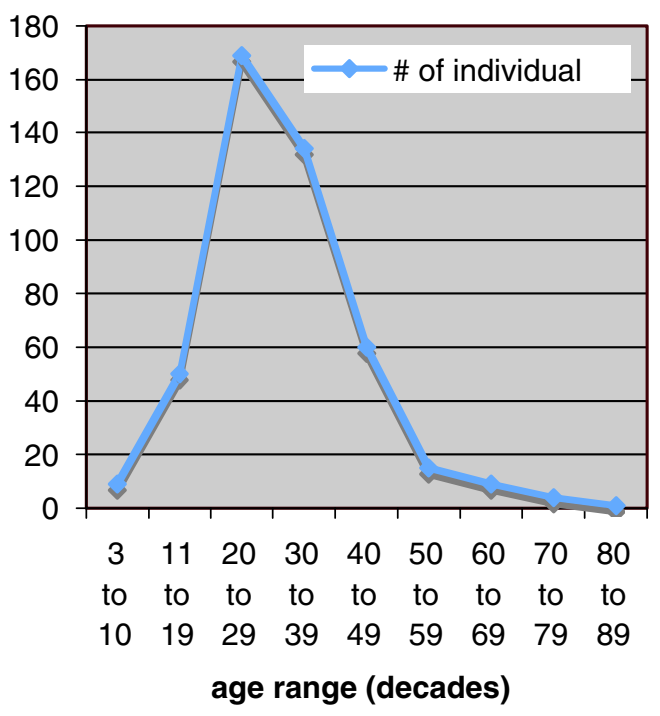

Fig. 3 Age distribution of patients

studies into 9 categories to facilitate data analysis (Table 1). These include:

1. Abdominal ultrasound: Intra-abdominal organs not including the right upper quadrant. This category consisted of splenic ultrasound and evaluation for suspected intra-abdominal mass.

2. Pelvic ultrasound-gravid: Studies that were done in pregnant patients or those thought to be pregnant. These studies were done primarily for pregnancy staging. Among the 132 ultrasounds performed, progression of pregnancy was determined in 113 , incidental finding of twins in 11, intrauterine fetal demise in 6 , and 2 cases in which women who appeared gravid were found to not be pregnant.

3. Renal ultrasound: Studies that looked specifically at the kidneys and bladder. Normal exams accounted for 35 of the 54 studies. Among the abnormal studies, hydronephrosis (9) and renal cysts (4) were the most common findings.

4. Pelvic ultrasound-non-gravid: Transabdominal ultrasound studies of the female pelvic organs in presumed non-pregnant women. Of 120 studies, 32 were noted as normal. The most common abnormal findings were ovarian cysts, uterine fibroids, unexpected pregnancy, and ovarian mass.

5. Prostate ultrasound: Studies showed several cases of enlarged prostate $(6 / 10)$, one prostatic mass, and three normal exams.

6. Right upper quadrant ultrasound: Studies of the gallbladder, liver, and Morrison's pouch. Normal exams were noted in 25 of 49 studies. Hepatomegaly was noted in eight cases, cholecystitis in four, symp- 
Table 1 Distribution of ultrasound study types
$R U Q$ right upper quadrant

\begin{tabular}{lllll}
\hline Study type & No. of studies & \% normal & \% abnormal & \% of total exams \\
\hline Pelvic-gravid & 132 & 86 & 14 & 24.1 \\
Abdominal (not RUQ) & 124 & 44 & 56 & 22.7 \\
Pelvic-non-gravid & 120 & 27 & 73 & 21.9 \\
Renal & 54 & 60 & 40 & 9.9 \\
Right upper quadrant & 49 & 51 & 49 & 9.0 \\
Prostate & 10 & 30 & 70 & 1.8 \\
Cardiac & 5 & 60 & 40 & 0.9 \\
Soft tissue & 2 & 0 & 100 & 0.4 \\
"Ultrasound exam" & 51 & 100 & 0 & 9.3
\end{tabular}

tomatic cholelithiasis in three, and previously unrecognized ascites in two.

7. Ultrasound exam: This category represents ultrasound exams that were not categorized by the practitioners with results of "normal exam" noted in the log. This category accounted for $9.3 \%$ of the total studies performed.

8. Cardiac ultrasound: Studies to evaluate for pericardial effusion. Two of the five performed were positive.

9. Soft tissue ultrasound: Studies to evaluate for suspected abscess versus solid skin mass. Both of the examinations performed identified solid masses.

\section{Discussion}

Ultrasound and tropical disease

Although the availability of ultrasound is generally poor throughout the developing world [2, 5], a number of tropical diseases and pathological conditions commonly found in the developing world are well suited to ultrasound evaluation and diagnosis. For example, WHO has supported the establishment of an international standardized classification of ultrasonographic findings in echinococcosis (hydatidosis) $[12,13]$. The ultrasound findings in cases of hepatosplenic schistosomiasis are often specific enough to obviate the need for liver biopsy [12]. Complications of urinary schistosomiasis are also readily evaluated using ultrasound [14]. Amebic liver abscesses are well visualized using ultrasound, and in the appropriate clinical setting ultrasound findings may be sufficient to make the diagnosis [12]. Parasitic diseases including lymphatic filariasis and biliary ascariasis have also been successfully evaluated using ultrasound [12].

Cardiac ultrasound has several diagnostic applications that are particularly suited to the developing world. Strang reported particular utility of echocardiography in evaluating patients for tuberculous pericarditis in South Africa and in guiding pericardiocenteses in these cases. Among the 1,004 echo recordings he made, 682 patients were ultimately diagnosed with this disease entity, earning it the moniker "Transkei heart" [15].

\section{Ultrasound training}

The effectiveness of ultrasonography as a diagnostic tool is dependent on the training of the ultrasonographer. WHO established standards in ultrasound training in 1998 that recommended a minimum of 200 obstetric and abdominal examinations prior to being considered competent to interpret scans [16]. With the more recent development of emergency ultrasound as a distinct diagnostic approach, emphasis has been placed on goal-oriented ultrasound examinations that address specific questions such as "Is there free fluid in the peritoneum?" or "Is there an intrauterine pregnancy?". Although no credentialing standard for emergency ultrasound training exists, the American College of Emergency Physicians (ACEP) published guidelines in 2001 that often serve as a basis for training curricula [17]. In addition to requirements for hands-on experience in each of the six core ultrasound examinations, ACEP recommended a minimum $16-\mathrm{h}$ initial didactic training course.

Due to geographic constraints, the training that we provided during our feasibility study in Lugufu was not developed to follow ACEP guidelines. No credentialed emergency sonographer would be available going forward, lack of Internet access precluded transmission of images and over-reading, and no "formal" ultrasound or computed tomography (CT) scan results would be available to serve as comparison studies. We did, however, emphasize goaloriented ultrasound and the importance of not over-relying on negative exams to rule out pathological conditions. Hoyer and colleagues reported success conducting nearly exclusively hands-on training over a 2 -week period in The Gambia and pointed out that local practical training automatically adapts the "curriculum" to local clinical circumstances [18].

Our team hopes to return again to Lugufu to further reinforce the teaching from our previous visits. Ideally we 
would be able to implement a system in which consultation via electronic transmission of images or footage captured by the refugee camp providers could be conducted. Unfortunately, at this time Internet access is not readily available at Lugufu.

\section{Assessment of use}

Since the donation of the ultrasound machine to the TRC camp at Lugufu, the machine has been perceived as a useful diagnostic tool. From the data, it appears that a large portion of exams was performed on women of childbearing age. This is in part explained by the age distribution of the refugee population which is skewed towards the left due to high fertility rates and short life expectancy. The machine proved to be most useful for female pelvic and obstetric issues: distinguishing ovarian cysts from uterine fibroids, determining pregnancy, following progression of pregnancies, and identifying abnormal and twin pregnancies. Table 1 summarizes the distribution of ultrasound examinations performed.

\section{Limitations}

This study represents a feasibility effort to integrate emergency ultrasound into the health services of a large refugee camp and has several important limitations. An important challenge that became evident in the review of the Lugufu data is that of data integrity. As we reviewed the data we noted the quality of the data decreased over time. This presents a significant challenge when trying to further analyze the data. Obviously, the 51 ultrasound studies classified as simply "ultrasound exam" with "normal" results cannot be interpreted. Despite these limitations, there is a strong commitment from the TRC health care providers to keep records of their ultrasounds and share their data.

Given the operator-dependent nature of ultrasound, objectively assessing the skills of our trainees and developing a system to over-read images would have greatly increased our confidence that the machine was being used appropriately and effectively in medical decision making. A longer training period, more frequent visits to reinforce teaching, utilization of a practical and/or written exam to assess trainee skills, and transmission of images (by Internet or mail) would all be steps to address these shortcomings.

Finally, although our trainees confidently reported that the use of ultrasound improved their care for patients, no outcome data were measured during this project, so the impact of ultrasound on clinical care at Lugufu is uncertain.
The case for ultrasound in low-resource settings

The case for ultrasound in this and similar very lowresource settings is easy to make. Ultrasound is safe, portable, inexpensive relative to other diagnostic imaging modalities, requires a simple power supply, and minimal maintenance. The versatility and wide range of clinical applications for ultrasound make it the imaging modality best suited in these settings. Ultrasound-based practice could reduce the need for other more expensive equipment in low-resource settings [19]. Likewise, given the extremely low capacity referral chains that are available in most lowresource settings, the benefit of obtaining information from on-site ultrasound is magnified and can help obviate the need for referral or provide further justification for crossing the high threshold necessary to utilize referral services. In reflecting how the practicality and advantages of ultrasound make it superior to other imaging modalities in low-resource settings, one editorial noted "those who hold that ultrasound should be the first (and often only) investigation will have that view enhanced if the setting is Africa" [20].

WHO developed their BRS when ultrasound technology had hardly grown beyond its infancy. Initially, WHO and others clearly stated that ultrasound should be considered only after conventional radiography is available [7, 21]. More recently, the WHO Scientific Group on Clinical Diagnostic Imaging described three levels of imaging equipment considered to be appropriate technology in different health care settings. Level I was designed for the most basic clinic or hospital settings where no radiology department or specialist is locally available and includes ultrasound in addition to the WHO-BRS. We recommend that conventional $\mathrm{X}$-ray capacity not be a prerequisite to obtaining an ultrasound unit.

\section{Directions for further studies}

There are numerous opportunities to build on our effort with future studies. Specific inquiries into training protocols could help to establish an improved ultrasound curriculum that is most appropriate for the clinical and geographic settings involved. A quantitative analysis of the impact of on-site ultrasound on the local referral chain could help to demonstrate the economic and logistical impact of ultrasound in this setting. Studies carried out in Western settings, such as the impact of ultrasound availability on time to operating room in cases of ectopic pregnancy, could be duplicated in very low-resource settings to evaluate if these benefits translate outside of developed settings. Finally, qualitative research that assesses social and cultural perspectives on the introduction of a new technology such as ultrasound could improve implementation. 


\section{Conclusion}

Our feasibility study demonstrates the enthusiastic embrace of a portable ultrasound unit by the health care staff of a large refugee camp in the tropics and suggests that health care providers in austere settings can use portable ultrasound to facilitate the care of their patients. Furthermore, it demonstrates that a portable ultrasound unit can be a sustainable addition to the health care services in such a setting.

Conflicts of interest None.

\section{References}

1. de Geus A (1993) Scarcity of radiodiagnostic services in developing countries. Trop Geogr Med 45(3):97

2. Brederhoff J, Racoveanu NT (1982) Radiological services throughout the world. Diagn Imaging 51(3-4):121-133

3. Viamonte M, Martinez LO, Hanson G et al (1990) World Health Organization-basic radiographic system (WHO-BRS) unit: experimental clinical evaluation. Clin Radiol 41(5):321-325

4. Felkel S (1999) Ultrasound safety mechanical and thermal indices: a primer. J Diagn Med Sonogr 15(2):77-80

5. Palmer PE (1993) Imaging equipment for small hospitals. Trop Geogr Med 45(3):98-102

6. Leggat PA (1997) Basic radiological system: a case study in 'appropriate technology for better health'. Aust J Rural Health 5 (2):87-89

7. (1985) Future use of new imaging technologies in developing countries. Report of a WHO Scientific Group. World Health Organ Tech Rep Ser 723:1-67

8. Kurjak A, Breyer B (1986) The use of ultrasound in developing countries. Ultrasound Med Biol 12(8):611-621
9. Adler D (2003) Health services in the Lugufu 1 Refugee Camp: looking back six years after the deployment of the basic health care emergency response unit. Report to the IFRC, Geneva

10. Doehring-Schwerdtfeger E, Abdel-Rahim IM, Dittrich $M$ et al (1992) Ultrasonography as a diagnostic aid for a district hospital in the tropics. Am J Trop Med Hyg 46(6):727-731

11. Mets T (1991) Clinical ultrasound in developing countries. Lancet 337(8737):358

12. Richter J, Hatz C, Haussinger D (2003) Ultrasound in tropical and parasitic diseases. Lancet 362(9387):900-902

13. WHO Informal Working Group (2003) International classification of ultrasound images in cystic echinococcosis for application in clinical and field epidemiological settings. Acta Trop 85(2):253261

14. Houston S (1991) Ultrasound: appropriate technology for tropical field work. Trans R Soc Trop Med Hyg 85(3):321-323

15. Strang JI (1990) Echoes from the Third World - two-dimensional echocardiography in a developing country. S Afr Med J 77(2):8591

16. (1998) Training in diagnostic ultrasound: essentials, principles and standards. Report of a WHO Study Group. World Health Organ Tech Rep Ser 875:i-46; back cover

17. American College of Emergency Physicians (2001) ACEP policy statement: emergency ultrasound guidelines

18. Hoyer PF, Weber M (1997) Ultrasound in developing world Lancet 350(9087): 1330

19. Mindel S (1997) Role of imager in developing world. Lancet 350 (9075):426-429

20. (1990) Clinical ultrasound in developing countries. Lancet 336 (8725):1225-1226

21. Chaves OE (1983) How to introduce diagnostic ultrasound in medical institutions of limited resources. Ultrasound Med Biol (Suppl 2):87-90

David H. Adler is an Assistant Professor of Emergency Medicine and Community and Preventive Medicine at the University of Rochester in Rochester, NY. 